\title{
Atypical antipsychotics are associated with incident diabetes in older adults without schizophrenia or bipolar disorder
}

\section{(6) UNLOCKFD}

This paper is freely available online under the BMJ Journals unlocked scheme, see http:// ebmh.bmj.com/info/unlocked.dt

\section{QUESTION}

Question: Is atypical antipsychotic use associated with incident diabetes or hyperlipidaemia in older people without schizophrenia or bipolar disorder?

People: Medicare advantage or commercial managed healthcare plan enrolees aged 65 and above with no history of schizophrenia, bipolar disorder, diabetes (for the hyperlipidaemia study) or hyperlipidaemia (for the diabetes study) in the previous year. In the diabetes study, cases were 13075 people who initiated diabetes treatment between 2004 and 2008 (identification period), and controls were 65375 people who had not received diabetes treatment during this time. In the hyperlipidaemia study, cases were 63 829 people newly started on hyperlipidaemia medication, and controls were 63829 people who had not received hyperlipidaemia medication. Controls were matched to cases based on age, sex, health plan type and index date. The index date for cases was the date of first diabetes or hyperlipidaemia medication prescription fill. For controls, the index date was a randomly selected date in the identification period.

Setting: USA; from 2003 to 2008.

Risk factors: Atypical antipsychotic exposure in the year prior to diabetes or hyperlipidaemia treatment initiation (preindex period), identified using pharmacy claims data. Medications considered as atypical antipsychotics were aripiprazole, clozapine, olanzapine, paliperidone, quetiapine, risperidone and ziprasidone. Participants taking atypical antipsychotics were stratified according to the drug prescribed, dose and the number of days of exposure during the year before the index date. Analyses were adjusted for the overall burden of comorbidities (assessed using the Charlson Comorbidity Index) during the preindex period, and specific comorbidities including diabetes (in the hyperlipidaemia study), hyperlipidaemia (in the diabetes study), hypertension, obesity, dementia, depression, anxiety and adjustment disorders. The hyperlipidaemia study additionally adjusted for stroke, coronary heart disease or ischaemic heart disease as indicators for cardiovascular disease.

Outcomes: Incident onset of treatment-dependent diabetes or hyperlipidaemia.

\section{METHODS \\ Design: Two case control studies. \\ Follow-up period: One year.}

\section{MAIN RESULTS}

During the preindex period, $1.3 \%$ of diabetes cases had been exposed to an atypical antipsychotic compared with $0.8 \%$ of controls (OR 1.32, 95\% CI 1.10 to 1.59). A greater overall burden of comorbidity, diagnosis or treatment of hyperlipidaemia or hypertension, and diagnosis of obesity or dementia were all associated with increased odds of initiating diabetes treatment, while an anxiety diagnosis was associated with decreased odds of initiating diabetes treatment. In the hyperlipidaemia study, $0.8 \%$ of cases had been exposed to an atypical antipsychotic during the preindex period compared with $1.0 \%$ of controls (OR $0.76,95 \%$ CI 0.67 to 0.87 ). Greater burden of comorbidity, diagnosis or treatment of diabetes, depression, obesity or cardiovascular disease during the preindex period was associated with increased odds of initiating hyperlipidaemia treatment. A diagnosis of dementia or adjustment disorders was associated with decreased odds of initiating hyperlipidaemia treatment.

\section{CONCLUSIONS}

In older adults, treatment with atypical antipsychotics for conditions other than schizophrenia and bipolar disorder is associated with increased odds of incident medication use for diabetes, and reduced odds of incident medication use for hyperlipidaemia.

\section{ABSTRACTED FROM}

Erickson SC, Le L, Zakharyan A, et al. New-onset treatment-dependent diabetes mellitus and hyperlipidemia associated with atypical antipsychotic use in older adults without schizophrenia or bipolar disorder. J Am Geriatr Soc 2012;60:474-9.

Correspondence to: Sara C Erickson, Sr. Outcomes Researcher, OptumRx, 2300 Main St. CA134-0404, Irvine, CA 92614, USA; sara.c.erickson@optum. comCorrespondence to: Sara C Erickson, Sr. Outcomes Researcher, OptumRx, 2300 Main St. CA134-0404, Irvine, CA 92614, USA; sara.c.erickson@optum.com

Sources of funding Not reported.

- Notes and References are published online at http://ebmh.bmj.com
E rickson et al used data from multiple insurance companies to examine the risk of diabetes mellitus and hyperlipidaemia in older adults without schizophrenia or bipolar disorder who received treatment with atypical antipsychotic agents. This is an important study as it highlights the risk of metabolic disturbances in older adults treated with these agents. It also provided evidence that the increased risk for diabetes is not associated with the psychiatric diagnosis, but more so with the atypical antipsychotic agents themselves. The findings contradict a previous, smaller retrospective study that found no increased risk for diabetes in older patients treated with these agents. ${ }^{1}$ The results seem to fit more with clinical observation, which contrasts previous reports.
While the claims data methods used in this study have its limitations, the large sample size and comparison group increases its strength. The lack of an association with these agents and hyperlipidaemia is not surprising. The risk of hyperlipidaemia associated with the atypical antipsychotic agents lies mostly in their effects on insulin resistance which can impair lipid metabolism primarily through lowering highdensity lipoprotein cholesterol and increasing triglycerides. ${ }^{2}$ However, these two lipid abnormalities are less likely to result in the diagnosis of hyperlipidaemia or the recommendation of a lipid lowering drug. Even more so, the first-line treatment for elevated triglycerides is commonly the recommendation of diet and $\omega-3$ fatty acid treatment such as fish oil and does not necessitate a prescription.
The results of this study should inform clinicians of the need for close monitoring for diabetes mellitus in patients treated with these agents. Though difference between agents was not observed, the study highlights the importance of monitoring all patients treated with atypical antipsychotic agents and that an advanced age is not protective from the metabolic effects

\section{Neelam Thakurathi, David C Henderson} Department of Psychiatry, Massachusetts General Hospital and Harvard Medical School, Boston, Massachusetts, USA

Competing interests None. 